\title{
Genome-wide identification and expression analysis of microRNA involved in small cell lung cancer via deep sequencing
}

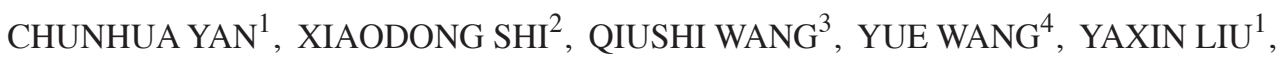 \\ XIAOFEI ZHANG ${ }^{1}$, YUANDI YANG ${ }^{1}$, FUZHEN LV $^{1}$ and YUXIA SHAO ${ }^{1}$ \\ Departments of ${ }^{1}$ Respiratory Medicine, ${ }^{2}$ Neurology and ${ }^{3}$ Thoracic Surgery, \\ the Second Affiliated Hospital of Harbin Medical University; ${ }^{4}$ Department of Occupational Health, \\ Public Health College, Harbin Medical University, Harbin 150081, P.R. China
}

Received October 28, 2013; Accepted May 22, 2014

DOI: $10.3892 / \mathrm{mmr} .2014 .2535$

\begin{abstract}
Small cell lung cancer is a major cause of mortality worldwide. microRNAs (miRNAs) are involved in various biological processes through regulating gene expression. In the present study, to identify the miRNAs involved in human small cell lung cancer at the genome-wide level, Solexa sequencing was employed to sequence two small RNA (sRNA) libraries from small cell lung cancer tissues (LC sRNA library) and the corresponding normal tissues (NT sRNA library). Deep sequencing of the two sRNA libraries identified a number of conserved miRNAs and differential expression analysis of these miRNAs revealed 81 miRNAs differentially expressed in small cell lung cancer, of which more than half were downregulated. The expression trends determined by sequencing were validated by reverse transcription-quantitative polymerase chain reaction analysis. The annotations for the targets of these miRNAs were predicted. This study provides valuable information for understanding the regulatory mechanisms of miRNAs involved in human small cell lung cancer.
\end{abstract}

\section{Introduction}

MicroRNAs (miRNAs) are small non-coding RNA molecules, $\sim 22$ nucleotides long, which are widely distributed in plants and animals $(1,2)$. The primary miRNA transcripts are cleaved by Drosha and Dicer enzymes to form mature miRNAs, which

Correspondence to: Mr. Fuzhen Lv or Mr. Yuxia Shao, Department of Respiratory MEdi, the Second Affiliated Hospital of Harbin Medical University, 246 Xuefu Road, Harbin 150081, P.R. China

E-mail: lvfuzhendoctor@gmail.com

E-mail: shaoyuxiadoctor@gmail.com

Abbreviations: miRNA, microRNA; sRNA, small RNA; RT-qPCR, reverse transcription-quantitative polymerase chain reaction; SCLC, small cell lung cancer; NSCLC, non-small cell lung cancer; EGFR, epidermal growth factor receptor

Key words: miRNA, small cell lung cancer, Solexa sequencing, RT-qPCR serve as posttranscriptional negative gene regulators by either cleaving mRNA or inhibiting translation $(3,4)$. Through such mechanisms, miRNAs are involved in various biological processes, including organ development, tissue differentiation, cell cycle regulation and cancer development (4-6).

Lung cancer is the predominant cause of cancer-related mortality worldwide and its pathogenesis is closely associated with tobacco smoking. Lung cancer is categorized into two main histological groups: Non-small cell lung cancer (NSCLC) and small cell lung cancer (SCLC). Regulation of miRNAs is closely associated with tumor initiation, promotion and progression (7). Recently, increasing evidence has demonstrated that miRNAs may be associated with lung cancer. A previous study revealed that an increase in hsa-miR-196a expression levels was a characteristic molecular change in NSCLC $(8,9)$, and that hsa-miRNA-196a promoted NSCLC cell proliferation and invasion via targeting HOXA5 (10). Overexpression of miR-200 was shown to reduce the expression levels of NSCLC prognostic biomarkers in H1299 and BEAS-2B cells (11). miR-449a, which inhibited migration and invasion through targeting $\mathrm{c}$-Met, was found to be downregulated in NSCLC tissues and cell lines (12). Reduced expression of miR-101 was associated with overexpression of $E Z H 2$ and exhibited tumor-suppressive functions in NSCLC (13). This evidence suggests that miRNAs are crucial in NSCLC; however, little is known regarding the functions and regulation of miRNAs in SCLC.

In the present study, Solexa sequencing was used to generate a large quantity of small RNA (sRNA) data for SCLC and corresponding normal tissues. Sequence analysis was employed to identify specific miRNAs associated with SCLC and these miRNAs were validated by reverse transcriptionquantitative polymerase chain reaction (RT-qPCR).

\section{Materials and methods}

Samples and total RNA isolation. The experimental specimens were obtained with informed consent between 2011 and 2012 from six patients at similar stages of SCLC. The SCLC tissues and corresponding normal tissues from healthy subjects were collected at the time of surgery and prior to chemotherapy at the Second Affiliated Hospital of Harbin Medical University 
(Harbin, China). This study was approved by the Hospitals' Ethical Review Committee. The isolated SCLC and corresponding normal tissues were harvested in liquid nitrogen and stored at $-80^{\circ} \mathrm{C}$. The total RNA was extracted from the SCLC tissues and controls using Trizol reagent (Invitrogen Life Technologies, Carlsbad, CA, USA).

sRNA library construction. Two sRNA libraries were constructed from the SCLC tissues (LC sRNA library) and the corresponding normal tissues (NT sRNA library) using methods described previously (14). Briefly, total RNA was purified by electrophoretic separation on a $15 \%$ Tris/Borate/EDTA urea denaturing PAGE gel (Sangon Biotech Co., Ltd., Shanghai, China) and sRNA molecules in the range of 15-30 nucleotides (ligated to adapters at the end of 5' and 3' sRNAs using T4 RNA ligase) were enriched (Takara Biotechnology Co., Ltd., Dalian, China). The adapter-ligated sRNAs were subsequently transcribed to complementary DNA (cDNA) by Super-Script II Reverse Transcriptase (Invitrogen Life Technologies) and then amplified by PCR. The PCR products were purified and recovered, then subjected to deep sequencing using an Illumina-Solexa 1G Genetic Analyzer (Illumina, Inc., San Diego, CA, USA) at the Beijing Genomics Institute (Shenzhen, China).

Differential expression analysis of miRNAs involved in $S C L C$. The raw readings generated by deep sequencing were analyzed through bioinformatics. These sequences were mapped to the human genome using the short oligonucleotide alignment program (15) with a perfect match. Sequences matching ribosomal (r)RNAs, small cytoplasmic (sc)RNAs, small nucleolar (sno)RNAs, snRNAs and transfer (t)RNAs in the NCBI GenBank (http://www.ncbi.nih.gov/GenBank/) database and Rfam (http://rfam.sanger.ac.uk/) database were discarded. The conserved miRNAs were predicted by aligning to miRBase 20.0 (http://www.mirbase.org/index.shtml); then the 187 conserved miRNAs (data not shown) were annotated by matching with miRBase 20.0. TargetScan (http://www. targetscan.org) was used for annotation of the targets of conserved miRNAs.

Differential expression levels of miRNAs were analyzed as determined by the sequence readings of LC and NT sRNA libraries. The abundance of miRNAs was normalized to one million (normalized expression $=$ actual miRNA count $/$ total count of clean readings $x 1,000,000)$. The ratio between the two sRNA libraries was calculated as follows: Ratio $=$ miRNA normalized readings in LC library/miRNA normalized readings in NT library. The P-values were calculated according to the equation in Fig. 1. When the ratio was $>1.5$-fold or $<0.67$-fold, and $\mathrm{P}<0.05$, the miRNAs were considered to be up- or downregulated respectively in SCLC.

Validation of differential expression levels of miRNAs by $R T$ - $q P C R$. sRNAs $(<200 \mathrm{nt})$ were isolated from the SCLC tissues (LC sRNA library) and the normal tissues using a mirVana miRNA Isolation kit (Ambion, Carlsbad, CA, USA). The tissues used for validation were the same as tissues used for the deep sequencing. The sRNAs were polyadenylated by poly(A) polymerase using the Poly(A) Tailing kit (Ambion) and then cDNA was synthesized with a Quant reverse kit

$$
\begin{aligned}
& P(x \mid y)=\left(\frac{N_{2}}{N_{1}}\right) \frac{(x+y) !}{x ! y !\left(1+\frac{N_{2}}{N_{1}}\right)^{(x+y+1)}} \\
& C(y \leq y \min \mid x)=\sum_{y=0}^{y \leq y \min } \mathrm{p}(y \mid x) \\
& D(y \geq y \max \mid x)=\sum_{y \geq y \max }^{\infty} \mathrm{p}(y \mid x)
\end{aligned}
$$

Figure 1. Equation for the P-value for the differential expression of the normal tissue (NT) and small cell lung cancer tissue (LC) micro (mi)RNAs. N1, total number of readings in the NT short RNA library; N2, total number of readings in the LC short RNA library, $\mathrm{x}$, number of readings for an miRNA in the control library; $y$, number of readings for an miRNA in the LC sRNA library

(Tiangen, Beijing, China) (15). RT-qPCR was performed using an ABI 7500 Fast Real-time PCR machine (Applied Biosystems, Foster City, CA, USA) using an SYBR Premix Ex TaqTM kit (Takara Bio, Inc., Shiga, Japan) in a 20- $\mu 1$ reaction volume, containing $2 \mu \mathrm{l}$ diluted cDNA, $200 \mathrm{nM}$ of each primer and 1X PCR Master mix. The amplification conditions were provided by Takara Bio Inc.. The expression levels of miRNAs were normalized to those of the U6 sRNA. Three replicates were analyzed for each sample. The primers for the validated miRNAs are shown in Table I.

Statistical analysis. The correlation analysis between the miRNA expression profile was conducted using bivariate correlation in SPSS software (SPSS, Inc., Chicago, IL, USA). P-values were used to reflect the significance of miRNA differential expression between these two sRNA libraries. $\mathrm{P}<0.05$ indicated a statistically significant difference.

\section{Results}

Analysis of $s R N A s$. To identify the responsive miRNAs in the SCLC patients, two sRNA libraries were constructed from the SCLC tissues (LC sRNA library) and the normal tissues (NT sRNA library), generating 25.1 million (LC) and 24.6 million (NT) raw readings. These sRNAs were classified into five categories: rRNAetc (rRNA, scRNA, snoRNA, snRNA and tRNA), known miRNAs (miRNAs in miRBase 20.0), exon, intron and unknown sRNA (Fig. 2). The results revealed that the proportion of total known miRNA readings decreased from $31.96 \%$ (NT) to $28.63 \%$ (LC) in response to SCLC, suggesting that the known miRNAs may be important in SCLC. However, the percentage of total rRNAetc readings increased from $23.87 \%$ (NT) to $26.86 \%$ (LC), implying that rRNAetc may exert certain functions in SCLC. Little difference was identified in the percentages of introns and unknown sRNAs between SCLC and normal tissue.

Identification of miRNAs in SCLC. Those miRNAs with a ratio of $>1.5$-fold or $<0.67$-fold between the two libraries, and $\mathrm{P}<0.05$ (Table II), were regarded as differentially expressed miRNAs in SCLC. A total of 81 differentially expressed 
Table I. Primers used for reverse transcription-polymerase chain reaction analysis.

Member

hsa-miR-21-5p

hsa-miR-22-3p

hsa-miR-29a-3p

hsa-miR-29a-5p

hsa-miR-30a-3p

hsa-miR-30a-5p

hsa-miR-34a-3p

hsa-miR-34a-5p

hsa-miR-99a-3p

hsa-miR-99a-5p

hsa-miR-100-3p

hsa-miR-100-5p

hsa-miR-101-5p

hsa-miR-126-5p

hsa-miR-134-3p

hsa-miR-139-3p

hsa-miR-141-5p

hsa-miR-143-3p

hsa-miR-145-5p

hsa-miR-148a-3p

hsa-miR-152-5p

hsa-miR-182-5p

hsa-miR-183-3p

hsa-miR-185-5p

hsa-miR-192-3p

hsa-miR-195-3p

hsa-miR-205-3p

hsa-miR-205-5p

hsa-miR-218-5p

hsa-miR-221-5p

hsa-miR-223-3p

hsa-miR-224-3p

hsa-miR-498

hsa-miR-557

hsa-miR-623

hsa-miR-1233-5p

Poly(T) adapter

Reverse primer

U6 small RNA
microRNA sequence (5'-3')

${ }^{\mathrm{a}} \mathrm{V}=\mathrm{A}, \mathrm{G}, \mathrm{C} ; \mathrm{N}=\mathrm{A}, \mathrm{T}, \mathrm{G}, \mathrm{C}$.

miRNAs were identified. The abundance of these miRNAs varied between 4 and 4,343 readings. Of these, 18 corresponding miRNA*s were identified, including hsa-miR-29a-3p and hsa-miR-29a-5p, and hsa-miR-30a-3p and hsa-miR-30a-5p (miRNA* is another mRNA that derives from the same hairpin structure as that of the original miRNA, and is complementary to the original miRNA). Notably, the expression levels of these 18 miRNAs were found to be associated with the corresponding

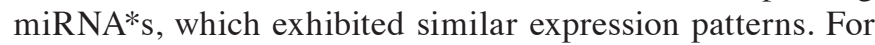

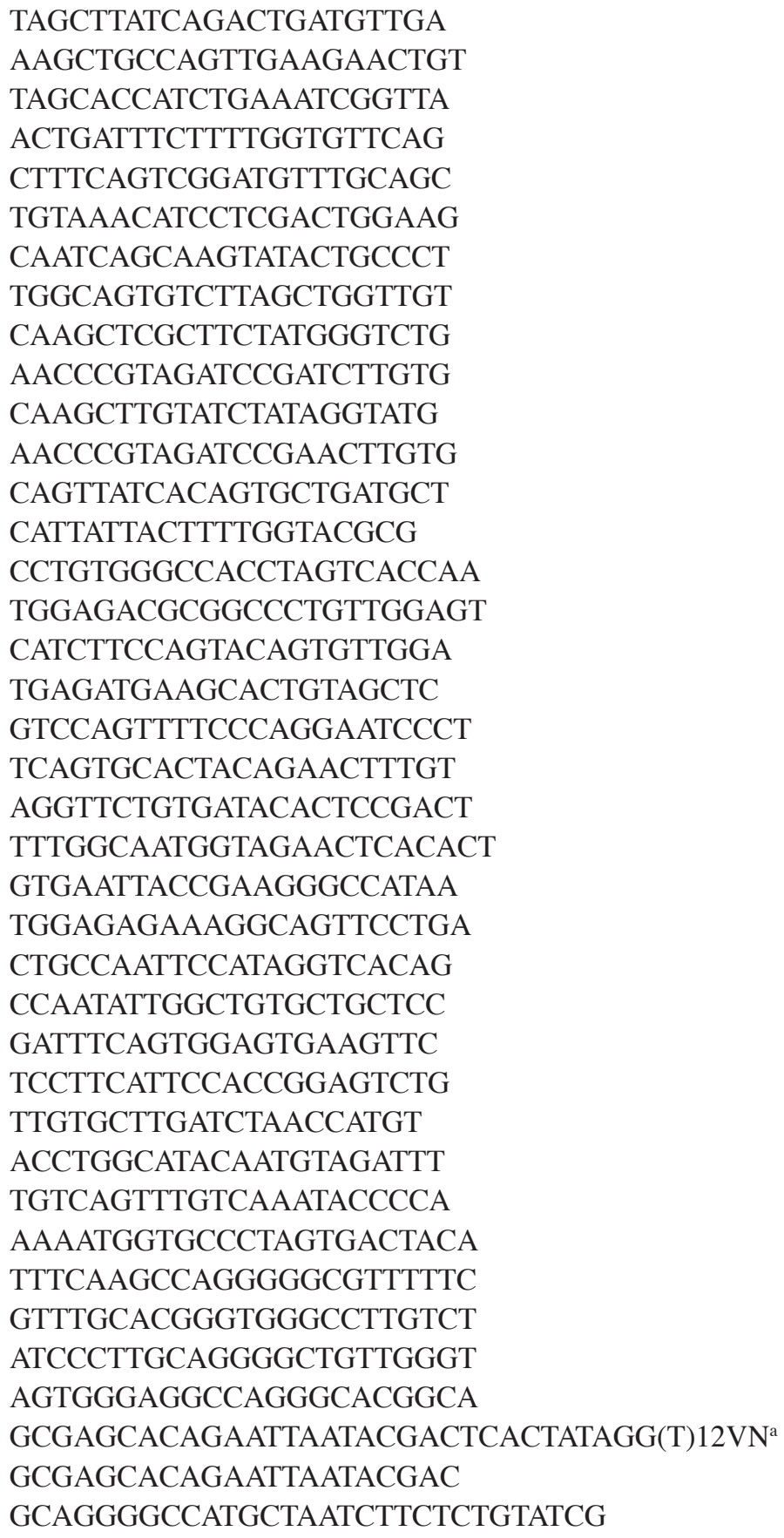

TAGCTTATCAGACTGATGTTGA

CTTTCAGTCGGATGTTTGCAGC

TGTAAACATCCTCGACTGGAAG

CAAGCTCGCTTCTATGGGTCTG

AACCCGTAGATCCGATCTTGTG

CAAGCTTGTATCTATAGGTATG

AACCCGTAGATCCGAACTTGTG

CAGTTATCACAGTGCTGATGCT

CATTATTACTTTTGGTACGCG

CCTGTGGGCCACCTAGTCACCAA

TGGAGACGCGGCCCTGTTGGAGT

CATCTTCCAGTACAGTGTTGGA

TGAGATGAAGCACTGTAGCTC

GTCCAGTTTTCCCAGGAATCCCT

TCAGTGCACTACAGAACTTTGT

AGGTTCTGTGATACACTCCGACT

TTTGGCAATGGTAGAACTCACACT

GTGAATTACCGAAGGGCCATAA

TGGAGAGAAAGGCAGTTCCTGA

CTGCCAATTCCATAGGTCACAG

CCAATATTGGCTGTGCTGCTCC

GATTTCAGTGGAGTGAAGTTC

TCCTTCATTCCACCGGAGTCTG

TTGTGCTTGATCTAACCATGT

TGTCA example, hsa-miR-146a-3p was downregulated and hsa-miR146a-5p was also downregulated, implying that the expression of miRNA*s followed the corresponding miRNAs. A total of 34 miRNAs were upregulated in SCLC and the remainder were downregulated. The most notable changes occurred in hsa-miR$520 \mathrm{c}-3 \mathrm{p}$, which had the greatest reduction in expression levels in SCLC with a ratio of 0.12 (LC/NT), and hsa-miR-148b-3p, which exhibited the greatest increase with 7.39-fold more readings sequenced in LC than in NT. These marked alterations 


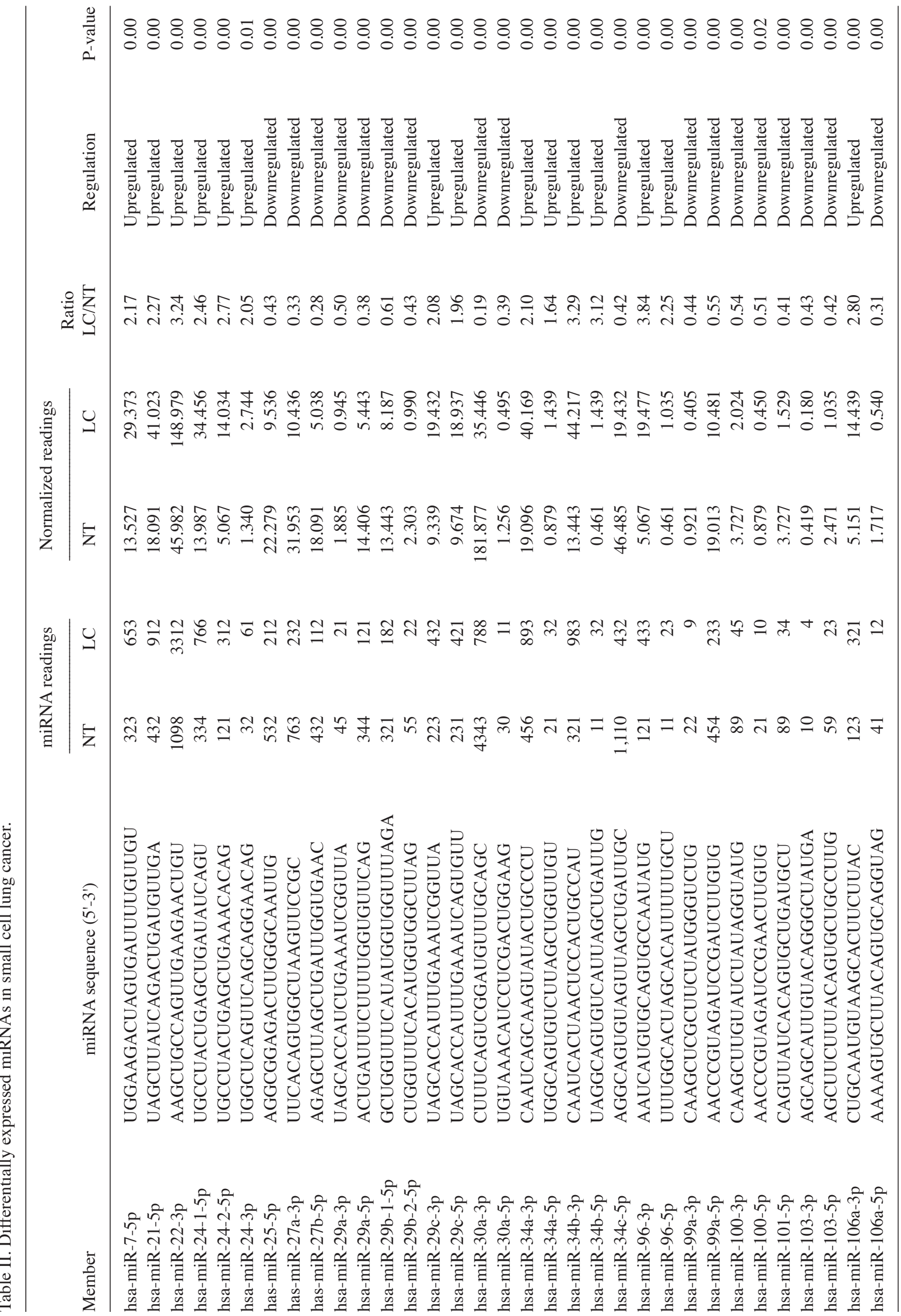




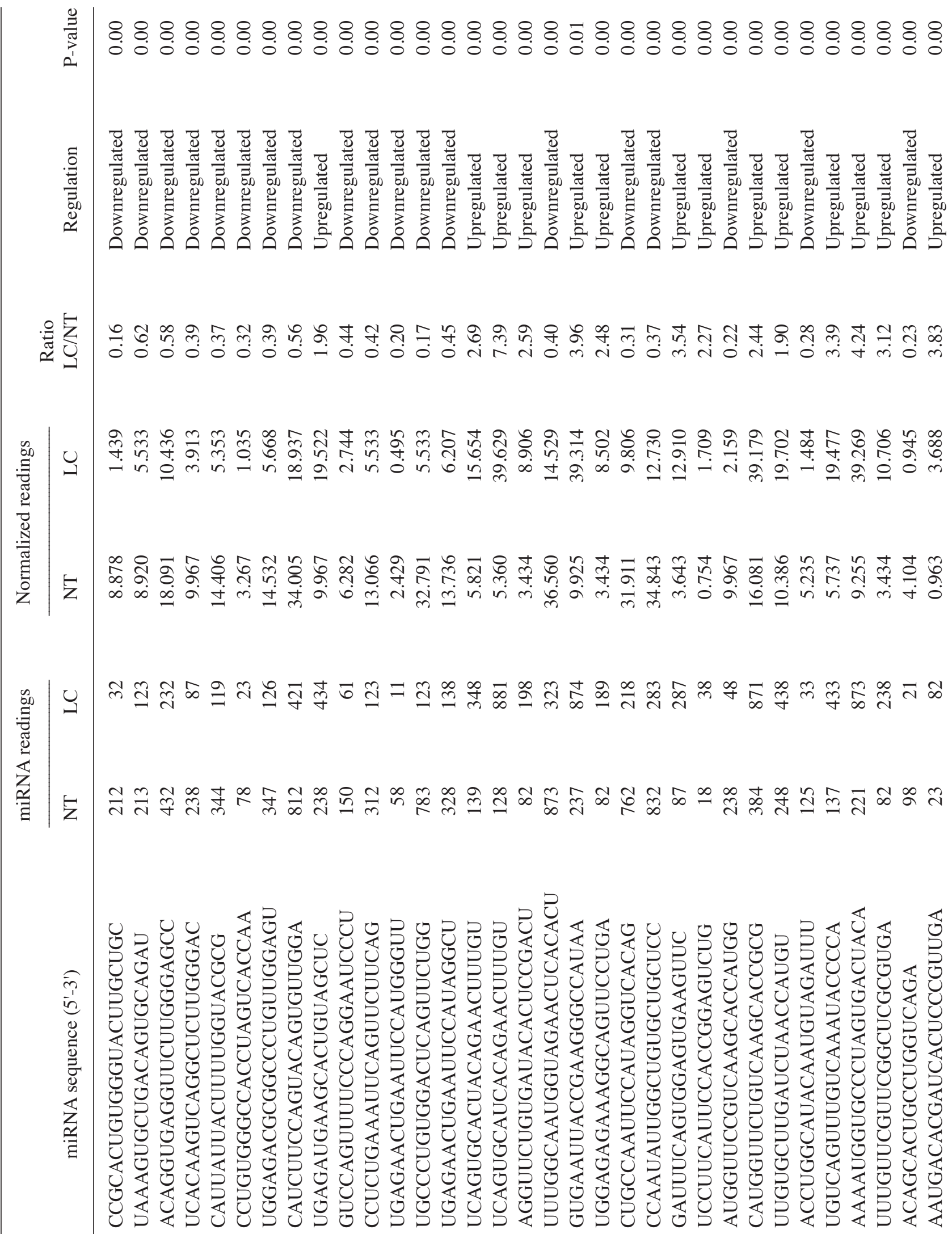

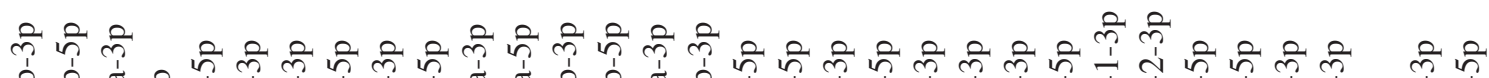
bे bै

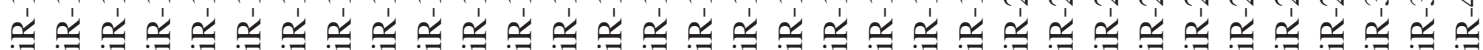

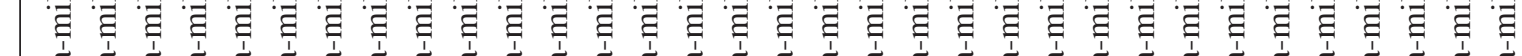

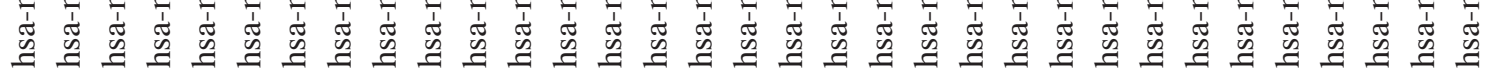




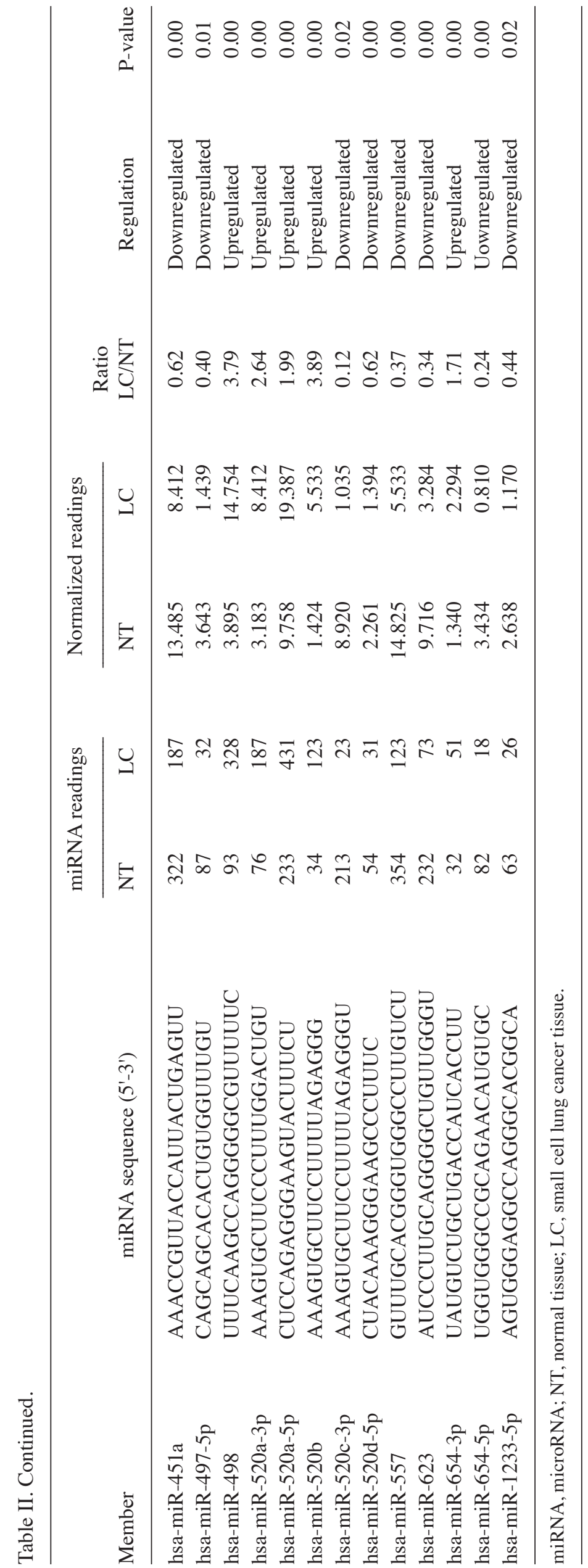




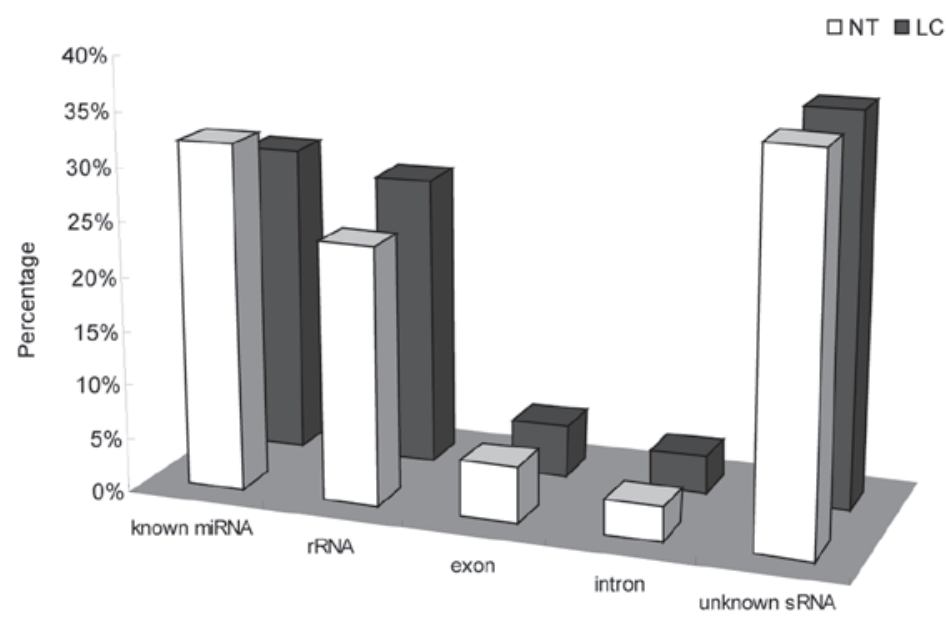

Figure 2. Distribution of different small (s)RNA annotation categories in small cell luncer cancer (LC) and normal tissue (NT) sRNA libraries.
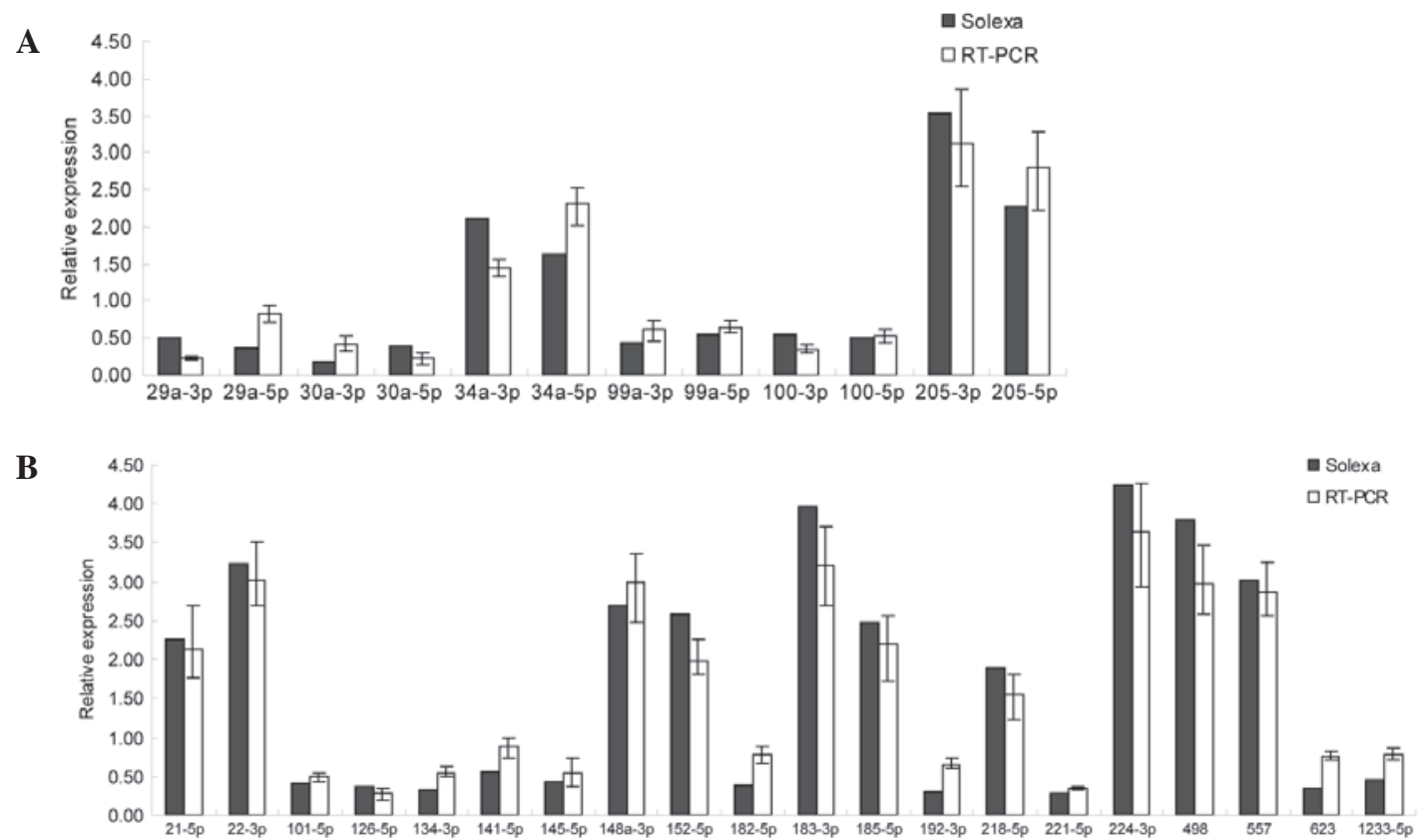

Figure 3. Validation of differential expression microRNAs extracted from small cell lung cancer tissues (LC) and normal tissues (NT) by reverse transcription-quantitative polymerase chain reaction (RT-qPCR). The expression levels of micro (mi)RNAs were normalized to the level of U6 short (s)RNA in the two sRNA libraries. The miRNA expression levels of normal tissues were used as a control and expression levels were arbitrarily set to 1 .

in expression levels, suggest that miRNAs are crucial in the response to SCLC.

$R T-q P C R$ validation. To validate the expression levels of the miRNAs involved in SCLC, RT-qPCR was performed on 36 miRNA sequences from the SCLC tissues and the corresponding normal tissues (Fig. 3). Of these 36 miRNAs, 6 miRNAs and the corresponding miRNA*s were identified, including hsa-miR-29a-3p and hsa-miR-29a-5p, and hsa-miR30a-3p and hsa-miR-30a-5p (Fig. 2A). This indicates that the mature miRNA and corresponding miRNA* exhibit similar expression patterns and are important in SCLC. The results revealed similar abundance profiles with Solexa sequencing and RT-qPCR analysis; they indicated that hsa-miR-21-5p was upregulated in the SCLC tissues. However, a few differences regarding the ratio $(\mathrm{LC} / \mathrm{NT})$ between $\mathrm{RT}-\mathrm{qPCR}$ and Solexa sequencing were identified; for example, the ratio of hsa-miR224-3p was 4.24 with sequencing data, but the RT-qPCR ratio was only 3.64 , possibly since different data normalization protocols of the two methods were provided. The sequencing was normalized to the whole abundance of all miRNAs sequenced by Solexa, while the RT-qPCR was normalized to the expression levels of U6 sRNA.

Annotation of the targets of miRNAs involved in SCLC. The targets of the miRNAs involved in SCLC were annotated by the TargetScan database. The functions of the targets of these miRNAs were involved in organ development, tissue differentiation, cell apoptosis and defense, signal transduction and the electron transfer chain. For example, the target of hsa-miR-29 encodes vascular endothelial growth factor A, which has various effects, including mediating increased vascular 
Table III. Targets of differentially expressed microRNAs in small cell lung cancer.

Member

hsa-miR-7

hsa-miR-21

hsa-miR-22

hsa-miR-24

has-miR-25

hsa-miR-29

hsa-miR-30

hsa-miR-34

hsa-miR-96

hsa-miR-99

hsa-miR-101

hsa-miR-103

hsa-miR-106

hsa-miR-125

hsa-miR-126

hsa-miR-139

hsa-miR-141

hsa-miR-143

hsa-miR-145

hsa-miR-146

hsa-miR-148/152

hsa-miR-182

hsa-miR-183

hsa-miR-192

hsa-miR-205
Target proteins

UBX domain protein 2B, spermatogenesis associated 2, phosphoinositide-3-kinase, catalytic, $\Delta$ polypeptide, ubiquilin 4, SH2 domain containing 5

Zinc finger protein $367, \mathrm{G}$ protein-coupled receptor 64, PHD finger protein 14, polybromo 1, vinculin, retinitis pigmentosa 2 (X-linked recessive)

Glutamate receptor, metabotropic 5 , coiled-coil domain containing 67 , fucosyltransferase $9(\alpha(1,3)$ fucosyltransferase), $\mathrm{H} 3$ histone, family $3 \mathrm{~B}$

Calcitonin receptor, mannose-P-dolichol utilization defect 1, kinase suppressor of ras 2, CKLF-like

MARVEL transmembrane domain containing 4, spermatogenesis associated, serine-rich 2-like

CD69 molecule, folliculin interacting protein 1 , actin, $\alpha$, cardiac muscle 1; F-box and WD repeat domain containing 7; aspartate $\beta$-hydroxylase

PIK3R1, Vascular endothelial growth factor A, PIK3RZ

MAPKS, NRG3, KRAS, PIK3CD, RARB, KRAS, CCNEZ, ITGA6, peroxisome proliferator-activated receptor $\gamma$, coactivator $1 \beta$, makorin ring finger protein 3

Cyclin E, p53; hyperpolarization activated cyclic nucleotide-gated potassium channel 3, family with sequence similarity 76 , member A; neuron navigator 3

Leucine-rich repeats and calponin homology domain containing 2; adenylate kinase 3; spindlin 1; dihydrolipoamide S-acetyltransferase

THAP domain containing, apoptosis associated protein 2; kelch repeat and BTB (POZ) domain containing 8; ependymin related protein 1 (zebrafish)

transportin 1 ; family with sequence similarity 108 , member $\mathrm{C} 1$; family with sequence similarity 108 , member C1; FLJ20160 protein

Dicer 1, ribonuclease type III; forkhead box P1; HIV-1 Rev binding protein; eukaryotic translation initiation factor 5; armadillo repeat containing 1

Carnitine O-octanoyltransferase; zinc finger with KRAB and SCAN domains 1; RAB22A, member RAS oncogene family; cytochrome $b$ reductase 1

StAR-related lipid transfer domain containing 13; zinc finger protein 792; SH3 domain and tetratricopeptide repeats 2; zinc finger and SCAN domain containing 29

ITGA6, CRK, SPRED1; epidermal growth factor-like domain 7; protein tyrosine phosphatase, non-receptor type 9; low density lipoprotein receptor-related protein 6; F-box protein 33

TATA element modulatory factor 1; USP6 N-terminal like; T-box 1; early B-cell factor 1; protein prenyltransferase $\alpha$ subunit repeat containing 1; S phase cyclin A-associated protein in the ER

Transmembrane protein 170B; zinc finger E-box binding homeobox 2; RAN binding protein 6; zinc finger RNA binding protein; protein kinase, cAMP-dependent, catalytic, $\beta$

Solute carrier family 30 (zinc transporter), member 8 ; vasohibin 1 ; homeodomain interacting protein kinase 2; development and differentiation enhancing factor-like 1

Epidermal growth factor receptor, IGF-1R; family with sequence similarity 108, member C1; SLIT-ROBO

Rho GTPase activating protein 2; ATP-binding cassette, sub-family E (OABP), member 1; tripartite motif-containing 2

Zinc finger protein 826; TNF receptor-associated factor 6; zinc finger and BTB domain containing 2; neuro-oncological ventral antigen 1 ; chemokine binding protein 2

Cholecystokinin B receptor; ATPase, $\mathrm{H}^{+}$transporting, lysosomal accessory protein 2; oxysterol binding protein-like 11; chloride channel 6

Regulator of G-protein signaling 17; microphthalmia-associated transcription factor; ARP2 actin-related protein 2 homolog (yeast); microfibrillar-associated protein 3; neurocalcin delta

VIL2-coding-protein Ezrin; A kinase anchor protein (gravin) 12; phosphatidylinositol glycan anchor biosynthesis, class X; neurotrophic tyrosine kinase, receptor, type 2; profilin 2; SLAIN motif family, member 1

IKAROS family zinc finger 2 (Helios); IKAROS family zinc finger 2 (Helios); poly(A) binding protein, cytoplasmic 4 (inducible form); dihydrolipoamide branched chain transacylase E2

RAB11 family interacting protein 1 (class I); RAB11 family interacting protein 1 (class I); cell division cycle 2-like 6 (CDK8-like); AP2 associated kinase 1; lysophosphatidylcholine acyltransferase 1 
Table III. Continued.

Member

Target proteins

hsa-miR-218

Chromosome 3 open reading frame 70; solute carrier family 1 (glial high affinity glutamate transporter), member 2; glucuronic acid epimerase; one cut homeobox 2; stress-associated endoplasmic reticulum protein 1

hsa-miR-221 Sorting nexin 4; regulator of G-protein signaling 6; osteopetrosis associated transmembrane protein 1; v-kit Hardy-Zuckerman 4 feline sarcoma viral oncogene homolog

hsa-miR-223 F-box and WD repeat domain containing 7; myosin VB; adenomatous polyposis coli; ras homolog gene family, member B; solute carrier family 4, sodium bicarbonate cotransporter, member 4

hsa-miR-375 TSC1, Phosphoinositide-dependent kinase-1, SOCS4, SPRY3, PRKX; solute carrier family 16, member 2 (monocarboxylic acid transporter 8); short stature homeobox 2; RAS, dexamethasone-induced 1

hsa-miR-383 NCK-associated protein 1; striatin, calmodulin binding protein 3; solute carrier family 35 (UDP-N-acetylglucosamine transporter), member A3; mal, T-cell differentiation protein 2

hsa-miR-425 Nuclear fragile X mental retardation protein interacting protein 2; family with sequence similarity 133, member B; forkhead box J3; sterile $\alpha$ motif and leucine zipper containing kinase AZK

hsa-miR-451a Tuberous sclerosis 1; chromosome 11 open reading frame 30; chromosome 11 open reading frame 30; GATA zinc finger domain containing $2 \mathrm{~B}$

hsa-miR-498 Chromosome X open reading frame 1; chromosome 9 open reading frame 5; CD2-associated protein; glutamate receptor, ionotrophic, AMPA 3

hsa-miR-520 Family with sequence similarity 102, member B; DIRAS family, GTP-binding RAS-like 2; sodium channel, voltage-gated, type III, $\beta$; homeodomain interacting protein kinase 2; YTH domain family, member 3

hsa-miR-623 Myotubularin related protein 7; acyl-CoA synthetase medium-chain family member 2A; adaptor-related protein complex 3, $\mu$ subunit; septin 11; ring finger protein 169

hsa-miR-654 Sorbin and SH3 domain containing 1; calcium channel, voltage-dependent, T type, $\alpha 1 \mathrm{I}$ subunit; transducin $(\beta)$-like 1X-linked; pregnancy-associated plasma protein A, pappalysin 1; pregnancy-associated plasma protein A, pappalysin 1; WD repeat domain 26

permeability, vasculogenesis and endothelial cell growth, promoting cell migration and inhibiting apoptosis $(17,18)$. The target of hsa-miR-375 encodes phosphoinositide-dependent kinase-1, which is important in the signaling pathways activated by several growth factors and hormones, including insulin (19). F-box and WD repeat domain-containing 7 encoded by hsa-miR-223 may regulate intestinal cell lineage commitment (20). The various functions of these targets implied that these responsive miRNAs exert crucial regulatory functions in SCLC. However, no functional annotations were identified for certain miRNAs, including hsa-miR-185 and hsa-miR-224 (Table III).

\section{Discussion}

SCLC is a leading cause of cancer-related morality worldwide. There are numerous reports regarding the roles of miRNAs in NSCLC $(10,12,21,22)$, however, little is known regarding the regulatory functions of miRNAs in SCLC. In the present study two sRNA libraries were constructed from SCLC and normal tissues. Sequence analysis and RT-qPCR validation identified 81 miRNAs differentially expressed in SCLC. This may provide useful information for improving the diagnosis, prevention and treatment of this disease. Notably, the expression levels of miRNA*s were associated with the corresponding miRNAs and revealed similar expression patterns. This may be due to miRNAs and the corresponding miRNA*s being derived from the same precursor (23). In addition, more than half the miRNAs associated with SCLC were downregulated and the total readings of known miRNAs were reduced (Fig. 2), implying that the corresponding targets become activated and trigger defense mechanisms against illness. However, further studies are required to characterize the miRNAs involved in SCLC.

Only a few studies have been published regarding the miRNAs involved in SCLC. Miko et al (24) employed microarray and RT-qPCR analyses to determine the miRNA expression profile in primary SCLC, and the result revealed that at least 24 miRNAs were differentially expressed between the normal lung and primary SCLC tumor tissues. A previous study performed gene expression profiling of drug-resistant SCLC cells by combining miRNA and cDNA expression analyses, and identified 61 significantly differentially expressed miRNAs (25). However, in the present study, a relatively complete analysis of miRNAs involved in SCLC at a genome-wide level was performed and certain miRNAs were reported to be involved in SCLC for the first time, including hsa-miR451. In addition, there were a few differences in the results of these studies. For example, hsa-miR-223 was downregulated in the previous study (25) while upregulated in the present study in SCLC tissues; this may be due to different methods for profiling miRNAs. 
The functions of the targets of miRNAs involved in SCLC were diverse and revealed inhibitory roles in the regulation of the corresponding targets. In the present study, the expression levels of hsa-miR-34-c, hsa-miR-126 and hsa-miR-145 were downregulated, while the expression levels of hsa-miR-183 was upregulated (Table II). The targets of hsa-miR-34, cyclin E and p53, are associated with the cell cycle and tumor formation $(26,27)$; the low expression of hsa-miR-34 may result in upregulation of cyclin E and p53, which is crucial in the development of SCLC. The epidermal growth factor receptor (EGFR) encoded by the target of hsa-miR-145 may inhibit cancer growth (28); the downregulation of hsa-miR-145 in SCLC may generate an increase in the expression levels of EGFR and thus promote cancer growth. The targets of hsa-miR-126 encode epidermal growth factor-like domain 7 (EGFL7), which is involved in cellular responses, such as cell migration and blood vessel formation (29). The downregulation of hsa-miR-126 may activate EGFL7 and advance tumor growth in vivo. The VIL2-coding-protein Ezrin, a known target of hsa-miR-183 is involved in migration and invasion (30); overexpression of hsa-miR-183 may repress VIL2-coding-protein Ezrin and inhibit migration and invasion of lung cancer cells, perhaps since defense mechanisms against illness have been initiated. Therefore, the evidence suggests that these identified miRNAs are important in SCLC.

In conclusion, 81 miRNAs involved in SCLC have been identified via deep sequencing and the profiles for a subset of miRNAs were validated by RT-qPCR. The functions for the target genes of these miRNAs were analyzed. These findings contribute to the understanding of the function of posttranscriptional regulation of miRNA in SCLC development and progression, which is essential for improving the diagnosis, prevention and treatment of this disease.

\section{References}

1. Bartel DP: MicroRNAs: genomics, biogenesis, mechanism, and function. Cell 116: 281-297, 2004.

2. Carrington JC and Ambros V: Role of microRNAs in plant and animal development. Science 301: 336-338, 2003.

3. Bartel DP: MicroRNAs: target recognition and regulatory functions. Cell 136: 215-233, 2009.

4. Kosik KS: MicroRNAs and cellular phenotypy. Cell 143: 21-26, 2010.

5. Alvarez-Garcia I and Miska EA: MicroRNA functions in animal development and human disease. Development 132: 4653-4662, 2005.

6. Inui M, Martello G and Piccolo S: MicroRNA control of signal transduction. Nat Rev Mol Cell Biol 11: 252-263, 2010.

7. Calin GA and Croce CM: MicroRNA signatures in human cancers. Nat Rev Cancer 6: 857-866, 2006.

8. Hui AB, Shi W, Boutros PC, et al: Robust global micro-RNA profiling with formalin-fixed paraffin-embedded breast cancer tissues. Lab Invest 89: 597-606, 2009.
9. Sun M, Liu XH, Li JH, Yang JS, et al: MiR-196a is upregulated in gastric cancer and promotes cell proliferation by downregulating p27(kip1). Mol Cancer Ther 11: 842-852, 2012.

10. Liu XH, Lu KH, Wang KM, et al: MicroRNA-196a promotes non-small cell lung cancer cell proliferation and invasion through targeting HOXA5. BMC Cancer 12: 348, 2012.

11. Pacurari M, Addison JB, Bondalapati N, et al: The microRNA-200 family targets multiple non-small cell lung cancer prognostic markers in H1299 cells and BEAS-2B cells. Int J Oncol 43: 548-560, 2013.

12. Luo W, Huang B, Li Z, et al: MicroRNA-449a Is downregulated in non-small cell lung cancer and inhibits migration and invasion by targeting c-Met. PLoS One 8: e64759, 2013.

13. Zhang JG, Guo JF, Liu DL, Liu Q and Wang JJ: MicroRNA-101 exerts tumor-suppressive functions in non-small cell lung cancer through directly targeting enhancer of zeste homolog 2 . J Thorac Oncol 6: 671-678, 2011.

14. Hafner M, Landgraf $\mathrm{P}$, Ludwig $\mathrm{J}$, et al: Identification of microRNAs and other small regulatory RNAs using cDNA library sequencing. Methods 44: 3-12, 2008.

15. Shi R and Chiang VL: Facile means for quantifying microRNA expression by real-time PCR. Biotechniques 39: 519-525, 2005.

16. Li R, Li Y, Kristiansen K and Wang J: SOAP: short oligonucleotide alignment program. Bioinformatics 24: 713-714, 2008.

17. Mackenzie F and Ruhrberg C: Diverse roles for VEGF-A in the nervous system. Development 139: 1371-1380, 2012.

18. Stockmann C, Doedens A, Weidemann A, et al: Deletion of vascular endothelial growth factor in myeloid cells accelerates tumorigenesis. Nature 456: 814-818, 2008.

19. Yamada T, Katagiri H, Asano T, et al: Role of PDK1 in insulin-signaling pathway for glucose metabolism in 3T3-L1 adipocytes. Am J Physiol Endocrinol Metab 282: E1385-E1394, 2002.

20. Sancho R, Jandke A, Davis H, et al: F-box and WD repeat domain-containing 7 regulates intestinal cell lineage commitment and is a haploinsufficient tumor suppressor. Gastroenterology 139: 929-941, 2010.

21. Yanaihara N, Caplen N, Bowman E, et al: Unique microRNA molecular profiles in lung cancer diagnosis and prognosis. Cancer Cell 9: 189-198, 2006.

22. Wang XC, Wang W, Zhang ZB, et al: Overexpression of miRNA-21 promotes radiation-resistance of non-small cell lung cancer. Radiat Oncol 8: 146, 2013.

23. Allen E, Xie Z, Gustafson AM, et al: Evolution of microRNA genes by inverted duplication of target gene sequences in Arabidopsis thaliana. Nat Genet 36:1282-1290, 2004.

24. Miko E, Czimmerer Z, Csánky E, et al: Differentially expressed microRNAs in small cell lung cancer. Exp Lung Res 35: 646-664, 2009.

25. Guo L, Liu Y, Bai Y, Sun Y, Xiao F and Guo Y. Gene expression profiling of drug-resistant small cell lung cancer cells by combining microRNA and cDNA expression analysis. Eur J Cancer 46: 1692-1702, 2010.

26. He L, He X, Lim LP, et al: A microRNA component of the p53 tumour suppressor network. Nature 447: 1130-1134, 2007.

27. Liu X, Sempere LF, Galimberti F, Freemantle SJ, et al: Uncovering growth-suppressive MicroRNAs in lung cancer. Clin Cancer Res 15: 1177-1183, 2009.

28. Zhong M, Ma X, Sun C and Chen L: MicroRNAs reduce tumor growth and contribute to enhance cytotoxicity induced by gefitinib in non-small cell lung cancer. Chem Biol Interact 184: 431-438, 2010.

29. Sun Y, Bai Y, Zhang F, Wang Y, et al: miR-126 inhibits non-small cell lung cancer cells proliferation by targeting EGFL7. Biochem Biophys Res Commun 391: 1483-1489, 2010.

30. Wang G, Mao W and Zheng S: MicroRNA-183 regulates Ezrin expression in lung cancer cells. FEBS Lett 582: 3663-3668, 2008. 